\title{
$q$-DEPENDENT CORRELATION FUNCTIONS AND DIELECTRIC PERMITTIVITIES OF DKDP AND DADP TYPE CRYSTALS. INFLUENCE OF EXTERNAL PRESSURE
}

\author{
A.P.Moina \\ Institute for Condensed Matter Physics \\ of the Ukrainian National Academy of Sciences \\ 1 Svientsitskii St., UA-290011 Lviv-11, Ukraine
}

Received March 6, 1997

\begin{abstract}
Within the four-particle cluster approximation, we obtain an equation for the $\boldsymbol{q}$-dependent deuteron pair correlation functions of DKDP and DADP crystals to which external hydrostatic and uniaxial $\sigma_{3}$ pressure is applied. Their wavevector, temerature and pressure dependences are studied. Expressions for the dielectric permittivities of the strained crystals are derived. It is shown that under the proper choice of theory parameters, the obtained results agree well with the available experimental data.
\end{abstract}

\section{Introduction}

Lately, a great attention has been paid to investigations of external pressure effects in KDP-type crystals. In our recent papers [1-6] we developed an approach (based on the model $[7,8]$ ) which allowed one to describe the pressure dependences of the transition temperature, dielectric and thermal properties of deuterated ferroelectrics and antiferroelectrics of DKDP and DADP type. The calculations were carried out within the four particle cluster approximation. It allowed us to take into account the strong shortrange correlations between deuterons adequately. A good description of the available experimental data was obtained, and some predictions concerning the effects of uniaxial pressure $\sigma_{3}$ on the considered responses of the crystals were made. Further experimental studies of hydrostatic and, especially, uniaxial pressure influence on these crystals are required to verify our predictions and determine the theory parameters more precisely.

In understanding the nature of phase transitions in KDP type crystals and studying their internal structure, the thermal neutron scattering technique is extremely useful. Since the calculation of neutron cross-sections may be reduced to calculation of appropriate correlation functions [9], to study the latters becomes exceedingly important. Besides that, analytical expressions for correlation functions enable one to calculate the corresponding dielectric susceptibility tensor.

Deuteron $\boldsymbol{q}$-dependent correlation functions of unstrained DKDP and DADP crystals have been calculated in a few papers. Thus, in [10] the equation for the $\boldsymbol{q}$-dependent correlation functions of DKDP and DADP crystals was suggested. No consistent derivation of it was given. The calculations were carried out for the case of $\boldsymbol{q}=0$ only. The obtained results correspond to the cluster approximation only at $T>T_{\mathrm{C}}$. 
The method of calculation of the $\boldsymbol{q}$-dependent correlation functions of paraelectric DKDP for a model with short-range interactions alone was proposed in [11]. In the four-particle cluster approximation, the dielectric susceptibility as a function of wavevector of some directions was calculated, and some numerical results were presented.

The consistent procedure for calculation of $\boldsymbol{q}$-dependent correlation functions of the model of a DKDP crystal with both short-range and long-range interactions (the latter being taken into account in the mean field approximation) in the cluster approximation was proposed in [12]. An equation of the Ornstein-Zernike type for pair correlation functions was derived both for $T>T_{\mathrm{C}}$ and $T<T_{\mathrm{C}}$. Components of the dielectric susceptibilty tensor were calculated for some directions of wavevector.

In this work, following the method of [12], we derive an equation for pair $\boldsymbol{q}$-dependent correlation functions of DKDP and DADP crystals to which external hydrostatic and uniaxial $\sigma_{3}$ pressure is applied. In Section 2, we suggest the unified model of strained DKDP and DADP crystals. In Section 3, the equations for pair correlation functions are derived. In Section 4, the expressions for dielectric permittivities are presented. Results of numerical calculations are given in Section 5 .

\section{The model}

We consider a system of deuterons moving on $\mathrm{O}-\mathrm{D}$... O bonds in a crystal of $\mathrm{KD}_{2} \mathrm{PO}_{4}$ (DKDP) or $\mathrm{ND}_{4} \mathrm{D}_{2} \mathrm{PO}_{4}$ (DADP) type. The primitive cell of such a crystal is composed of two neighbouring $\mathrm{PO}_{4}$ tetrahedra together with four hydrogen bonds attached to one of them (" $A$ " type tetrahedra). Hydrogen bonds going to another (" $B$ " type) tetrahedron belong to four nearest structural elements surrounding it. An external hydrostatic $\left(\sigma_{\mathrm{h}}\right)$ or uniaxial stress $\left(\sigma_{3}\right)$, which does not lower the system symmetry

$$
\sigma_{\mathrm{h}}=(-p,-p,-p), \quad \sigma_{3}=(0,0,-p)
$$

and electric field $E_{i}(i=1,2,3)$ directed along the crystallographic axes $a$, $b, c$ are applied.

The Hamiltonian of the system has the following form [7]

$$
\begin{aligned}
& \hat{H}= \frac{\bar{v} N}{2} \sum_{i j} c_{i j}^{(0)} \varepsilon_{i} \varepsilon_{j}-\frac{1}{2} \sum_{q q^{\prime}} \sum_{f f^{\prime}} J_{f f^{\prime}}\left(q q^{\prime}\right) \frac{\sigma_{q f}}{2} \frac{\sigma_{q^{\prime} f^{\prime}}}{2}+H_{\text {short }}-\sum_{q f} h_{q f}^{i} \frac{\sigma_{q f}}{2},(2.2) \\
& H_{\text {short }}=\left\{\frac{1}{2} \sum_{f f^{\prime}} V_{f f^{\prime}} \frac{\sigma_{q_{f} f}}{2} \frac{\sigma_{q_{f^{\prime}} f^{\prime}}}{2}+\Phi \frac{\sigma_{q_{1} 1}}{2} \frac{\sigma_{q_{2} 2}}{2} \frac{\sigma_{q_{3} 3}}{2} \frac{\sigma_{q_{4}}}{2}\right\} \times \\
& \times \sum_{q_{1}, q_{2} q_{3}, q_{4}}\left\{\delta_{\mathbf{R}_{q_{1}}, \mathbf{R}_{q_{2}}} \delta_{\mathbf{R}_{q_{1}}, \mathbf{R}_{q_{3}}} \delta_{\mathbf{R}_{q_{1}}, \mathbf{R}_{q_{4}}}+\delta_{\mathbf{R}_{q_{1}}+\mathbf{r}_{2}, \mathbf{R}_{q_{2}}} \delta_{\mathbf{R}_{q_{1}}+\mathbf{r}_{3}, \mathbf{R}_{q_{3}}} \delta_{\mathbf{R}_{q_{1}}+\mathbf{r}_{4}, \mathbf{R}_{q_{4}}}\right\} ; \\
& h_{q f}^{i}=\mu_{q f}^{i} E_{i} .
\end{aligned}
$$

Hamiltonian (2.3) describes short-range configurational interactions between deuterons near tetrahedra of "A" and "B" type; $\mathbf{r}_{f}$ is a relative position vector of a hydrogen bond in a cell. Two eigenvalues of Ising spin $\sigma_{q f}= \pm 1$ are assigned to two equilibrium positions of a deuteron on the $f$-th bond in the $q$-th unit cell. $c_{i j}^{(0)}$ are the "seed" elastic constants; $\varepsilon_{i}$ are 
the components of the strain tensor; $\bar{v}=v / k_{\mathrm{B}} ; v$ is the unit cell volume; $k_{\mathrm{B}}$ is the Boltzmann constant.

$$
V_{12}=V_{23}=V_{34}=V_{41}=V, \quad V_{13}=V_{24}=U,
$$

for ferroelectric crystals of DKDP type. Here

$$
\begin{aligned}
& V=-w_{1} / 2, \quad U=-\varepsilon+w_{1} / 2, \quad \Phi=4 \varepsilon-8 w+2 w_{1} ; \\
& \varepsilon=\varepsilon_{a}-\varepsilon_{s}, \quad w=\varepsilon_{1}-\varepsilon_{s}, \quad w_{1}=\varepsilon_{0}-\varepsilon_{s},
\end{aligned}
$$

$\varepsilon_{s}, \varepsilon_{a}, \varepsilon_{1}, \varepsilon_{0}$ are the energies of lateral, up-down, single-ionized and double ionized deuteron configurations, respectively. Let us note that the energy $\varepsilon_{s}$ is assumed to be the lowest.

For antiferroelectric crystals of DADP type

$$
\begin{aligned}
& V=\left(\varepsilon-w_{1}\right) / 2, \quad U=\left(\varepsilon+w_{1}\right) / 2, \quad \Phi=2 \varepsilon-8 w+2 w_{1} ; \\
& \varepsilon=\varepsilon_{s}-\varepsilon_{a}, \quad w=\varepsilon_{1}-\varepsilon_{a}, \quad w_{1}=\varepsilon_{0}-\varepsilon_{a},
\end{aligned}
$$

the energy of the lateral configurations $\varepsilon_{a}$ is the lowest. Within the proposed model the energies $\varepsilon, w$, and $w_{1}$ are assumed to be linear functions of strains $\varepsilon_{i}$

$$
\begin{aligned}
& \varepsilon=\delta_{11} \varepsilon_{1}+\delta_{12} \varepsilon_{2}+\delta_{13} \varepsilon_{3}+\varepsilon^{(0)}, \\
& w=\delta_{21} \varepsilon_{1}+\delta_{22} \varepsilon_{2}+\delta_{23} \varepsilon_{3}+w^{(0)}, \\
& w_{1}=\delta_{31} \varepsilon_{1}+\delta_{32} \varepsilon_{2}+\delta_{33} \varepsilon_{3}+w_{1}^{(0)} .
\end{aligned}
$$

We consider only diagonal components of the strain tensor $\varepsilon_{1}, \varepsilon_{2}, \varepsilon_{3}$, and neglect piezoelectric shear strains $\varepsilon_{4}, \varepsilon_{5}, \varepsilon_{6}$ arising when the respective electric fields $E_{1}, E_{2}$ and $E_{3}$ are applied (the piezomodules $d_{14}, d_{25}, d_{36}$ differ from zero). Therefore, the calculated dielectric susceptibilities will correspond to clamped $\left(\varepsilon_{i}=\right.$ const $)$ crystals.

$J_{f f^{\prime}}\left(q q^{\prime}\right)$ are the constants of long-range forces (dipole-dipole interactions and indirect interaction between deuterons through lattice vibrations), which we shall take into account in the mean field approximation. Let us note that if one formally expands the constants of the long-range interaction $J_{f f^{\prime}}\left(q q^{\prime}\right)$ in powers of strains up to the linear terms

$$
J_{f f^{\prime}}\left(q q^{\prime}\right)=\tilde{J}_{f f^{\prime}}^{(0)}\left(q q^{\prime}\right)+\sum_{j} \psi_{f f^{\prime}}^{j}\left(q q^{\prime}\right) \varepsilon_{j} ;
$$

then the obtained expressions for the mean fields

$$
æ_{q f}=\sum_{q^{\prime} f^{\prime}}\left[\tilde{J}_{f f^{\prime}}^{(0)}\left(q q^{\prime}\right)+\sum_{j} \psi_{f f^{\prime}}^{j}\left(q q^{\prime}\right) \varepsilon_{j}\right] \frac{\eta_{q^{\prime} f^{\prime}}^{(1)}}{2}+h_{q f}^{i},
$$

$\left(\eta_{q f}^{(1)} \equiv\left\langle\sigma_{q f}\right\rangle^{H}\right.$, averaging being carried out with the Hamiltonian (2.2)) coincide with those found in $[7,8]$ - the long-range interaction, taken into account in the mean field approximation, is renormalized by terms linear in the mean values of quasispins $\left\langle\sigma_{q f}\right\rangle^{H}$ and strains $\varepsilon_{i}$, - calculated considering only the lattice strains but neglecting the pressure dependence of the dipole moment of a hydrogen bond $\mu=e \delta(\delta$ is the $\mathrm{D}$-site distance on a O-D... bond). 
The pressure dependence of $\mu$ can be taken into account in the following way. According to $[13,14], \delta$ in KDP and DKDP crystals is a linear function of hydrostatic pressure. Assuming that the character of this dependence on hydrostatic and uniaxial $\sigma_{3}$ stress in DADP crystal and uniaxial stress in DKDP is the same and bearing in mind (2.1) we can write

$$
\delta=\delta_{0}+\delta_{1} p
$$

Expanding $\tilde{J}_{f f}$ in terms of pressure and considering the fact that it is proportional to $\mu^{2}$, we get

$$
\begin{aligned}
& \tilde{J}_{f f^{\prime}}^{(0)}\left(q q^{\prime}\right)=J_{f f^{\prime}}^{(0)}\left(q q^{\prime}\right)\left[1+2 \frac{\delta_{1}}{\delta_{0}} p\right] \\
& æ_{q f}=\sum_{q^{\prime} f^{\prime}}\left[J_{f f^{\prime}}^{(0)}\left(q q^{\prime}\right)\left[1+2 \frac{\delta_{1}}{\delta_{0}} p\right]+\sum_{j} \psi_{f f^{\prime}}^{j}\left(q q^{\prime}\right) \varepsilon_{j}\right] \frac{\eta_{q^{\prime} f^{\prime}}^{(1)}}{2}+h_{q f}^{i} .
\end{aligned}
$$

The parameters $\psi_{f f^{\prime}}\left(q q^{\prime}\right)$ for a given crystal are the same for all pressures which do not lower its symmetry, whereas the ratio $\delta_{1} / \delta_{0}$ is different for hydrostatic and uniaxial stresses.

The following calculations will be carried out in the cluster approximation. Considering the structure of the crystals, it is natural to divide the quasispin lattice into the four-particle clusters, vertices of which we choose in the centers of the $\mathrm{O}-\mathrm{D} \ldots \mathrm{O}$ bonds. As

$$
f^{f_{2}} \Delta_{q_{1} f_{1}}
$$

we denote an effective field acting on the spin $\sigma_{q_{1} f_{1}}$ from the side of its nearest neighbour $f_{2}$. Let us make an identical transformation (the long-range interactions have been taken into account in the mean field approximation)

$$
\begin{aligned}
& H=\frac{\bar{v} N}{2} \sum_{i j} c_{i j}^{(0)} \varepsilon_{i} \varepsilon_{j}+\frac{1}{2} \sum_{q f} \sum_{q^{\prime} f^{\prime}} J_{f f^{\prime}}\left(q q^{\prime}\right) \frac{\left\langle\sigma_{q f}\right\rangle^{H}}{2} \frac{\left\langle\sigma_{q^{\prime} f^{\prime}}\right\rangle^{H}}{2}+H^{\prime} \\
& H^{\prime}=H_{\text {short }}-\sum_{q f} \frac{\tilde{z}_{q f}}{\beta} \frac{\sigma_{q f}}{2}-\sum_{q} \sum_{f f^{\prime}} f^{\prime} \Delta_{q f} \frac{\sigma_{q f}}{2}=\sum_{q f} H_{q f}^{(1)}+\sum_{R} U_{R},
\end{aligned}
$$

where $H^{\prime}$ is the Hamiltonian of the so-called reference system, and

$$
\begin{aligned}
& H_{q f}^{(1)}=-\frac{\tilde{z}_{q f}}{\beta} \frac{\sigma_{q f}}{2} ; \\
& U_{R}=V\left[\frac{\sigma_{R 1}}{2} \frac{\sigma_{R 2}}{2}+\frac{\sigma_{R 2}}{2} \frac{\sigma_{R 3}}{2}+\frac{\sigma_{R 3}}{2} \frac{\sigma_{R 4}}{2}+\frac{\sigma_{R 4}}{2} \frac{\sigma_{R 1}}{2}\right]+ \\
& +U\left[\frac{\sigma_{R 1}}{2} \frac{\sigma_{R 3}}{2}+\frac{\sigma_{R 2}}{2} \frac{\sigma_{R 4}}{2}\right]+\Phi \frac{\sigma_{R 1}}{2} \frac{\sigma_{R 2}}{2} \frac{\sigma_{R 3}}{2} \frac{\sigma_{R 4}}{2}-\sum_{f \in R} \sum_{f^{\prime} \in \pi_{f} \in R} \frac{f^{\prime} \Delta_{R f}}{\beta} \frac{\sigma_{R f}}{2} .
\end{aligned}
$$

The summation in $\sum_{R}$ is carried out over clusters instead of lattice sites. Hereafter, it is implied that the clusters $R, R^{\prime}$ belong to the cell $q$, clusters $R_{1}, R_{1}^{\prime}$ belong to the cell $q_{1}$ etc; $\pi_{f}$ denotes the set of the nearest neighbours of the site $f$.

$$
\tilde{z}_{q f}=\beta\left[æ_{q f}-\sum_{f^{\prime} \in \pi_{f}} f^{\prime} \Delta_{q f}\right] .
$$


Let us define the generating function $\mathcal{F}$ of the reference system as

$$
\mathcal{F}=\ln \mathrm{Sp}^{-\beta H^{\prime}}=\ln \mathrm{Sp}\left\{\exp \left(-\beta \sum_{q f} H_{q f}^{(1)}\right) \exp \left(-\beta \sum_{R} U_{R}\right)\right\}
$$

Then the free energy of the crystal is equal to

$$
F=\frac{v \bar{N}}{2} \sum_{i j} c_{i j} \varepsilon_{i} \varepsilon_{j}+\frac{1}{2} \sum_{q f} \sum_{q^{\prime} f^{\prime}} J_{f f^{\prime}}\left(q q^{\prime}\right) \frac{\left\langle\sigma_{q f}\right\rangle^{H}}{2} \frac{\left\langle\sigma_{q^{\prime} f^{\prime}}\right\rangle^{H}}{2}-\frac{\mathcal{F}}{\beta} .
$$

Our goal is to calculate the correlation functions (cumulant averages of spin operators products, in particular, single-particle and pair ones) which are related to the free energy in the following way:

$$
\left\langle\sigma_{q_{1} f_{1}} \ldots \sigma_{q_{n} f_{n}}\right\rangle_{c}^{H}=\left(\frac{2}{\beta}\right)^{n} \frac{\delta}{\delta h_{q_{1} f_{1}}} \ldots \frac{\delta(-\beta F)}{\delta h_{q_{n} f_{n}}} .
$$

respectively, the correlation functions of the reference system are

$$
\left\langle\sigma_{q_{1} f_{1}} \ldots \sigma_{q_{n} f_{n}}\right\rangle^{c}=\left(\frac{2}{\beta}\right)^{n} \frac{\delta}{\delta æ_{q_{1} f_{1}}} \ldots \frac{\delta \mathcal{F}}{\delta æ_{q_{n} f_{n}}} .
$$

From (2.8)-(2.11) it follows that the single-particle correlation functions of the reference and general systems coincide $\left\langle\sigma_{q f}\right\rangle^{H}=\left\langle\sigma_{q f}\right\rangle$, whereas for the pair correlation functions the following relations hold

$$
\left\langle\sigma_{q_{1} f_{1}} \sigma_{q_{2} f_{2}}\right\rangle_{c}^{H}=\sum_{q_{3} f_{3}}\left\langle\sigma_{q_{1} f_{1}} \sigma_{q_{3} f_{3}}\right\rangle^{c}\left[\delta_{q_{3} q_{2}} \delta_{f_{3} f_{2}}-\frac{\beta}{4} \sum_{q_{4} f_{4}} J_{f_{3} f_{4}}\left(q_{3} q_{4}\right)\left\langle\sigma_{q_{3} f_{3}} \sigma_{q_{2} f_{2}}\right)^{c}\right]^{-1} .
$$

Let us introduce the notations

$$
\begin{aligned}
& \langle(\ldots)\rangle_{0} \equiv \frac{\operatorname{Sp}\left\{(\ldots) \exp \left(-\beta \sum_{q f} H_{q f}^{(1)}\right)\right\}}{\operatorname{Sp} \exp \left(-\beta \sum_{q f} H_{q f}^{(1)}\right)}, \\
& F_{q f}^{(1)} \equiv \ln \operatorname{Sp} \exp \left(-\beta H_{q f}^{(1)}\right)=\ln 2 \cosh \frac{\tilde{z}_{q f}}{2} .
\end{aligned}
$$

Then

$$
\mathcal{F}=\sum_{q f} F_{q f}^{(1)}+\ln \left\langle\exp \left[-\beta \sum_{R} U_{R}\right]\right\rangle_{0} .
$$

We restrict our consideration to the first order of the cluster expansion, that is

$$
\ln \left\langle\exp \left[-\beta \sum_{R} U_{R}\right]\right\rangle_{0} \simeq \sum_{R} \ln \left\langle\exp \left[-\beta U_{R}\right]\right\rangle_{0} .
$$

Then, the generating function $\mathcal{F}$ becomes

$$
\mathcal{F}=\sum_{R} \ln \operatorname{Sp} \exp \left[-\beta H_{R}^{(4)}\right]-\sum_{q f} F_{q f}^{(1)}=\sum_{R} F_{R}^{(4)}-\sum_{q f} F_{q f}^{(1)},
$$


where

$$
\begin{aligned}
& H_{R}^{(4)}=V\left[\frac{\sigma_{R 1}}{2} \frac{\sigma_{R 2}}{2}+\frac{\sigma_{R 2}}{2} \frac{\sigma_{R 3}}{2}+\frac{\sigma_{R 3}}{2} \frac{\sigma_{R 4}}{2}+\frac{\sigma_{R 4}}{2} \frac{\sigma_{R 1}}{2}\right]+ \\
& +U\left[\frac{\sigma_{R 1}}{2} \frac{\sigma_{R 3}}{2}+\frac{\sigma_{R 2}}{2} \frac{\sigma_{R 4}}{2}\right]+\Phi \frac{\sigma_{R 1}}{2} \frac{\sigma_{R 2}}{2} \frac{\sigma_{R 3}}{2} \frac{\sigma_{R 4}}{2}-\sum_{f \in R} \frac{z_{R f}}{\beta} \frac{\sigma_{R f}}{2}
\end{aligned}
$$

is the four-particle cluster Hamiltonian. Here we use the fact that since each site of the given lattice belongs to two neighbouring clusters then

$$
\sum_{q f}(\ldots)=\frac{1}{2} \sum_{R} \sum_{f \in R}(\ldots)
$$

The fields $z_{R f}$ are

$$
z_{R f}=\beta\left[æ_{R f}-\sum_{f^{\prime} \in \pi_{f} \notin R} f^{\prime} \Delta_{R f}\right]=\beta\left[æ_{R f}-\Delta_{R f}\right],
$$

the sum contains effective fields created by the neighbouring quasispins outside the cluster; for the lattice considered, $\Delta_{R f}$ is an effective field created by the whole neighbouring cluster $R^{\prime}$, which also contains the site $R f$. Let us note that

$$
\tilde{z}_{R f}=\beta\left[æ_{R f}-\sum_{f^{\prime} \in \pi_{f}} f^{f^{\prime}} \Delta_{R f}\right]=\beta\left[æ_{R f}-\Delta_{R^{\prime} f}-\Delta_{R f}\right],
$$

the field $\Delta_{R^{\prime} f}$ is created by the spins of the cluster $R$.

From the condition of a minimum of the generating function $\mathcal{F}$ with respect to $\Delta_{R f}$

$$
\frac{\partial \mathcal{F}}{\partial \Delta_{R f}}=0
$$

it follows that

$$
\frac{\partial F_{R}^{(4)}}{\partial \mathfrak{x}_{R f}}=\beta \frac{\partial F_{R}^{(4)}}{\partial z_{R f}}=\frac{\partial F_{R f}^{(1)}}{\partial æ_{R f}}=\beta \frac{\partial F_{R}^{(1)}}{\partial \tilde{z}_{R f}} ; \quad \frac{\partial F_{R^{\prime}}^{(4)}}{\partial æ_{R f}}=\frac{\partial F_{R^{\prime} f}^{(1)}}{\partial \mathfrak{x}_{R f}},
$$

and

$$
\left\langle\sigma_{R f}\right\rangle=\frac{2}{\beta} \frac{\partial F_{R}^{(4)}}{\partial æ_{R f}}=\frac{2}{\beta} \frac{\partial F_{R}^{(1)}}{\partial æ_{R f}},
$$

(provided that the site $f$ belongs to the clusters $R$ and $R^{\prime}$ ), that is, the mean values of spins, calculated with the single-particle Hamiltonian $H_{q f}^{(1)}$ and the four-particle one $H_{R}^{(4)}$, must coincide. The relations (2.21) comprise the system of equations for the unknown fields $\Delta_{R f}$.

If no electric field is applied, the quantities $z_{R f}$ are uniform, that is,

\section{DKDP}

\section{$\underline{\text { DADP }}$}

$$
\begin{aligned}
& z^{f} \equiv z_{R 1}=z_{R 2}=z_{R 3}=z_{R 4}=z_{R^{\prime} 1}=z_{R^{\prime} 2}=z_{R^{\prime} 3}=z_{R^{\prime} 4}= \\
& \quad=\beta\left[-\Delta+\nu_{c}(0) \eta^{(1)}\right],
\end{aligned}
$$

$$
z_{R}^{a}=-z_{R 1}=z_{R 2}=z_{R 3}=-z_{R 4}=\beta\left[-\Delta_{R}+\nu_{a}\left(\mathbf{k}_{Z}\right) \eta_{R}^{(1)}\right]=z_{R^{\prime}}^{a},
$$


where

$$
\begin{aligned}
& \nu_{a}(\mathbf{k})=\nu_{a}^{0}(\mathbf{k})\left[1+2 \frac{\delta_{1}}{\delta_{0}} p\right]+\sum_{i} \psi_{a i}(\mathbf{k}) \varepsilon_{i} \\
& \nu_{c}(0)=\nu_{c}^{0}(0)\left[1+2 \frac{\delta_{1}}{\delta_{0}} p\right]+\sum_{i} \psi_{c i}(0) \varepsilon_{i} \\
& \nu_{a}^{0}(\mathbf{k})=\frac{1}{4}\left[J_{11}^{(0)}(\mathbf{k})-J_{13}^{(0)}(\mathbf{k})\right] ; \quad \nu_{c}^{0}(0)=\frac{1}{4}\left[J_{11}^{(0)}(0)+2 J_{12}^{(0)}(0)+J_{13}^{(0)}(0)\right], \\
& \psi_{a i}(\mathbf{k})=\frac{1}{4}\left[\psi_{11}^{i}(\mathbf{k})-\psi_{13}^{i}(\mathbf{k})\right] ; \quad \psi_{c i}(0)=\frac{1}{4}\left[\psi_{11}^{i}(0)+2 \psi_{12}^{i}(0)+\psi_{13}^{i}(0)\right] . \\
& J_{f f^{\prime}}^{(0)}(\mathbf{k})=\sum_{\mathbf{R}_{q}-\mathbf{R}_{q^{\prime}}} J_{f f^{\prime}}^{(0)}\left(q q^{\prime}\right) \mathrm{e}^{-\mathbf{k}\left(\mathbf{R}_{q}-\mathbf{R}_{q^{\prime}}\right)}, \quad \psi_{f f^{\prime}}^{i}(\mathbf{k})=\sum_{\mathbf{R}_{q}-\mathbf{R}_{q^{\prime}}} \psi_{f f^{\prime}}^{i}\left(q q^{\prime}\right) \mathrm{e}^{-\mathbf{k}\left(\mathbf{R}_{q}-\mathbf{R}_{q^{\prime}}\right)},
\end{aligned}
$$

$\left(\mathbf{k}_{Z}=\left(\mathbf{b}_{1}+\mathbf{b}_{2}+\mathbf{b}_{3}\right) / 2, \mathbf{b}_{1}, \mathbf{b}_{2}, \mathbf{b}_{3}\right.$ are the basic vectors of the reciprocal lattice; the averages $\left\langle\sigma_{R f}\right\rangle$ are equal for both $R$ and $R^{\prime}$ clusters and obey the relations

\section{$\underline{\mathrm{DKDP}}$}

$$
\begin{gathered}
\eta^{(1)}=\eta_{R 1}^{(1)}=\eta_{R 2}^{(1)}=\eta_{R 3}^{(1)}=\eta_{R 4}^{(1)}=\eta_{R^{\prime} 1}^{(1)}=\eta_{R^{\prime} 2}^{(1)}=\eta_{R^{\prime} 3}^{(1)}=\eta_{R^{\prime} 4}^{(1)}, \\
\frac{\mathrm{DADP}}{\eta_{R}^{(1)}}=-\eta_{R 1}^{(1)}=\eta_{R 2}^{(1)}=\eta_{R 3}^{(1)}=-\eta_{R 4}^{(1)}=\eta^{(1)} \mathrm{e}^{i \mathbf{k}_{Z} \mathbf{R}_{q}}=\eta_{R^{\prime}}^{(1)}
\end{gathered}
$$

the factor $\mathrm{e}^{i \mathbf{k}_{Z} \mathbf{R}_{q}}= \pm 1$ denotes two sublattices of antiferroelectric DADP crystal.

The fields $z_{R f}$ are determined from the condition $(2.21)$. In the uniform case

\section{$\underline{\mathrm{DKDP}}$}

$$
z^{f}=\frac{1}{2} \ln \frac{1+\eta^{(1)}}{1-\eta^{(1)}}+\beta \nu_{c}(0) \eta^{(1)} ;
$$

\section{$\underline{\text { DADP }}$}

$$
z_{R}^{a}=z^{a} \mathrm{e}^{i \mathbf{k}_{Z} \mathbf{R}_{q}}=\left[\frac{1}{2} \ln \frac{1+\eta^{(1)}}{1-\eta^{(1)}}+\beta \nu_{a}\left(\mathbf{k}_{Z}\right) \eta^{(1)}\right] \mathrm{e}^{i \mathbf{k}_{Z} \mathbf{R}_{q}} .
$$

Let us now calculate the averages $\left\langle\sigma_{R f}\right\rangle$ and $\left\langle\sigma_{R f} \sigma_{R f^{\prime}}\right\rangle$ (the intracluster correlation functions), provided that (2.22)-(2.24) hold:

\section{$\underline{\mathrm{DKDP}}$}

$$
\begin{aligned}
& \eta^{(1)}=\frac{\sinh 2 z^{f}+2 b \sinh z^{f}}{D^{f}} \\
& \eta^{(2)}=\left\langle\sigma_{R 1} \sigma_{R 2}\right\rangle=\left\langle\sigma_{R 2} \sigma_{R 3}\right\rangle=\left\langle\sigma_{R 3} \sigma_{R 4}\right\rangle=\left\langle\sigma_{R 4} \sigma_{R 1}\right\rangle=\frac{\cosh 2 z^{f}-d}{D^{f}} \\
& \eta^{(2)^{\prime}}=\left\langle\sigma_{R 1} \sigma_{R 3}\right\rangle=\left\langle\sigma_{R 2} \sigma_{R 4}\right\rangle=\frac{\cosh 2 z^{f}-2 a+d}{D^{f}}
\end{aligned}
$$

$\underline{\mathrm{DADP}}$

$$
\eta_{R}^{(1)}=\frac{\sinh 2 z_{R}^{a}+2 b \sinh z_{R}^{a}}{D^{a}}
$$




$$
\begin{aligned}
& \eta^{(2)}=\left\langle\sigma_{R 1} \sigma_{R 4}\right\rangle=\left\langle\sigma_{R 2} \sigma_{R 3}\right\rangle==\frac{a-d+\cosh z_{R}^{a}-1}{D^{a}}, \\
& \eta^{(2)^{\prime}}=-\left\langle\sigma_{R 1} \sigma_{R 2}\right\rangle=-\left\langle\sigma_{R 3} \sigma_{R 4}\right\rangle=\frac{-a+d+\cosh z_{R}^{a}-1}{D^{a}}, \\
& \eta^{(2)^{\prime \prime}}=-\left\langle\sigma_{R 1} \sigma_{R 3}\right\rangle=-\left\langle\sigma_{R 2} \sigma_{R 4}\right\rangle=\frac{-a-d+\cosh z_{R}^{a}+1}{D^{a}} .
\end{aligned}
$$

The quantities $\eta^{(2)}$ are even functions of $z_{R}$ and do not depend on $q$;

$$
\begin{aligned}
& a=\exp [-\beta \varepsilon] ; \quad b=\exp [-\beta w] ; \quad d=\exp \left[-\beta w_{1}\right] ; \\
& D^{f}=\cosh 2 z^{f}+4 b \cosh z^{f}+2 a+d ; \\
& D^{a}=\cosh 2 z^{a}+4 b \cosh z^{a}+a+d+1 .
\end{aligned}
$$

Let us also introduce some notations for the cumulant averages which will be used in the following sections

$$
\begin{aligned}
& \frac{\text { DKDP }}{F_{0}^{f}}=1-\left(\eta^{(1)}\right)^{2}, \quad F_{1}^{f}=\eta^{(2)}-\left(\eta^{(1)}\right)^{2}, \quad F_{2}^{f}=\eta^{(2)^{\prime}}-\left(\eta^{(1)}\right)^{2}, \\
& \begin{aligned}
& \underline{\text {DADP}} \\
& F_{0}^{a}=1-\left(\eta_{R}^{(1)}\right)^{2}, \quad F_{1}^{a}=\eta^{(2)}-\left(\eta_{R}^{(1)}\right)^{2}, \\
& F_{2}^{a}=-\eta^{(2)^{\prime}}+\left(\eta_{R}^{(1)}\right)^{2}, \quad F_{3}^{a}=-\eta^{(2)^{\prime \prime}}+\left(\eta_{R}^{(1)}\right)^{2} .
\end{aligned}
\end{aligned}
$$

If no electric field is applied, the free energy of a crystal reads:

\section{DKDP}

$$
f^{f}=\frac{F^{f}}{N}=\frac{\bar{v}}{2} \sum_{i j} c_{i j}^{(0)} \varepsilon_{i} \varepsilon_{j}-2 w+2 \nu_{c}(0)\left[\eta^{(1)}\right]^{2}+2 T \ln \frac{2}{\left(1-\left[\eta^{(1)}\right]^{2}\right) D^{f}},
$$

$\underline{\mathrm{DADP}}$

$$
f^{a}=\frac{F^{a}}{N}=\frac{\bar{v}}{2} \sum_{i j} c_{i j}^{(0)} \varepsilon_{i} \varepsilon_{j}-2 w+\varepsilon+2 \nu_{a}\left(\mathbf{k}_{Z}\right)\left[\eta^{(1)}\right]^{2}+2 T \ln \frac{2}{\left(1-\left[\eta^{(1)}\right]^{2}\right) D^{a}} .
$$

The strains $\varepsilon_{i}$ and order parameter $\eta^{(1)}$ are determined from the conditions of the thermodynamic equilibrium

$$
\frac{1}{\bar{v}} \frac{\partial f}{\partial \eta^{(1)}}=0 ; \quad \frac{1}{\bar{v}} \frac{\partial f}{\partial \varepsilon_{i}}=-p_{i} .
$$

(with $\left.p_{i}(\mathrm{~h})=(p, p, p), \quad p_{i}(3)=(0,0, p)\right)$, namely, from the equations

\section{$\underline{\mathrm{DKDP}}$}

$$
\begin{aligned}
\eta^{(1)} & =\frac{1}{D^{f}}\left(\sinh 2 z^{f}+2 b \sinh z^{f}\right), \\
-p_{i} & =\sum_{j} c_{i j}^{(0)} \varepsilon_{j}-2 \frac{\delta_{2 i}}{\bar{v}}-\frac{2 \psi_{c i}}{\bar{v}}\left[\eta^{(1)}\right]^{2}+\frac{2}{\bar{v}} \frac{M_{i}^{f}}{D^{f}},
\end{aligned}
$$

DADP

$$
\begin{aligned}
\eta^{(1)} & =\frac{1}{D^{a}}\left(\sinh 2 z^{a}+2 b \sinh z^{a}\right), \\
-p_{i} & =\sum_{j} c_{i j}^{(0)} \varepsilon_{j}-\frac{2 \delta_{2 i}-\delta_{1 i}}{\bar{v}}-\frac{2 \psi_{a i}\left(\mathbf{k}_{Z}\right)}{\bar{v}}\left[\eta^{(1)}\right]^{2}+\frac{2}{\bar{v}} \frac{M_{i}^{a}}{D^{a}},
\end{aligned}
$$


where $M_{i}^{f}=4 b \delta_{2 i} \cosh z^{f}+2 a \delta_{1 i}+d \delta_{3 i}, M_{i}^{a}=4 b \delta_{2 i} \cosh z^{a}+a \delta_{1 i}+d \delta_{3 i}$.

As one can see from equations (2.30) and (2.31), the strains $\varepsilon_{i}$ are equal to zero at ambient pressure only if we assume the temperature dependence of the parameters $\delta_{2 i}$ in the form

$$
\delta_{2 i}^{+}=\frac{2 a \delta_{1 i}^{+}}{1+2 a}
$$

in the paraelectric phase of a DKDP crystal and

$$
\delta_{2 i}^{+}=\delta_{1 i}^{+} \frac{4 b+3 a+2}{2 a+4}
$$

in the paraelectric phase of a DADP crystal and in a more complicated form in the ordered phases. In order to keep $\delta_{2 i}$ constant and for the sake of simplicity we assumed that (2.32) and (2.33) are satisfied only at the transition points, that is,

$$
\begin{aligned}
& \frac{\mathrm{DKDP}}{\delta_{2 i}^{+}}=\frac{2 \exp \left(-\beta_{\mathrm{C}} \varepsilon\right) \delta_{1 i}^{+}}{1+2 \exp \left(-\beta_{\mathrm{C}} \varepsilon\right)}, \\
& \frac{\mathrm{DADP}}{\delta_{2 i}^{+}}=\delta_{1 i}^{+} \frac{4 \exp \left(-\beta_{\mathrm{N}} w\right)+3 \exp \left(-\beta_{\mathrm{N}} \varepsilon\right)+2}{2 \exp \left(-\beta_{\mathrm{N}} \varepsilon\right)+4},
\end{aligned}
$$

$\left(\beta_{\mathrm{C}}=1 / T_{\mathrm{C}}, \beta_{\mathrm{N}}=1 / T_{\mathrm{N}}\right)$. It means that at pressure of $0.001 \mathrm{kbar}$, small strains $\left(\sim 10^{-6}\right)$ exist at all temperatures except for the transition points.

\section{Equation for $q$-dependent correlation functions}

In this Section, following the procedure suggested in [12] for DKDP-type crystal at ambient pressure, we derive an equation of Ornstein-Zernike type for the $\boldsymbol{q}$-dependent pair correlation functions of strained DKDP and DADP crystals.

We need to calculate

$$
b_{f_{1} f_{2}}\left(R_{1} R_{2}\right) \equiv\left\langle\sigma_{R_{1} f_{1}} \sigma_{R_{2} f_{2}}\right\rangle^{c}=\frac{4}{\beta^{2}} \frac{\delta^{2} \mathcal{F}}{\delta æ_{R_{1} f_{1}} \delta \mathfrak{x}_{R_{2} f_{2}}} .
$$

Differentiating the expression for the single particle correlation function $(2.21)$

$$
b_{R_{1} f_{1}}=\left\langle\sigma_{R_{1} f_{1}}\right\rangle=2 \frac{\partial F_{R_{1}}^{(4)}}{\partial z_{R_{1} f_{1}}}=2 \frac{\partial F_{R_{1} f_{1}}^{(1)}}{\partial \tilde{z}_{R_{1} f_{1}}}
$$

with respect to $æ_{R_{2} f_{2}}$, we get:

$$
\left\langle\sigma_{R_{1} f_{1}} \sigma_{R_{2} f_{2}}\right\rangle^{c}=\frac{4}{\beta} \frac{\partial^{2} F_{R_{1} f_{1}}^{(1)}}{\partial \tilde{z}_{R_{1} f_{1}} \partial \tilde{z}_{R_{1} f_{1}}} \frac{\partial \tilde{z}_{R_{1} f_{1}}}{\partial æ_{R_{2} f_{2}}}=\frac{4}{\beta} \sum_{g \in R_{1}} \frac{\partial^{2} F_{R_{1}}^{(4)}}{\partial z_{R_{1} f_{1}} \partial z_{R_{1} g}} \frac{\partial z_{R_{1} g}}{\partial æ_{R_{2} f_{2}}}
$$


summation in (3.2) goes over all sites of the cluster $R_{1}$. Let us introduce the following notations:

$$
\begin{aligned}
& z_{f_{1} f_{2}}^{\prime}\left(R_{1} R_{2}\right) \equiv \frac{1}{\beta} \frac{\partial \bar{z}_{R_{1} f_{1}}}{\partial æ_{R_{2} f_{2}}} ; \quad \Delta_{f_{1} f_{2}}^{\prime}\left(R_{1} R_{2}\right) \equiv \frac{\partial \Delta_{R_{1} f_{1}}}{\partial æ_{R_{2} f_{2}}} . \\
& F_{f_{1} f_{2}}^{(4)}\left(R_{1}\right) \equiv 4 \frac{\partial^{2} F_{R_{1}}^{(4)}}{\partial z_{R_{1} f_{1}} \partial z_{R_{1} f_{2}}}=\left\langle\sigma_{R_{1} f_{1}} \sigma_{R_{1} f_{2}}\right)^{c}, \\
& F_{f_{1} f_{2}}^{(1)}\left(R_{1}\right) \equiv 4 \frac{\partial^{2} F_{R_{1} f_{1}}^{(1)}}{\partial \tilde{z}_{R_{1} f_{1}}^{2}} \delta_{f_{1} f_{2}}, \quad P_{f_{1} f_{2}}\left(R_{1}\right)=\left[\hat{F}^{(4)}\left(R_{1}\right)\right]_{f_{1} f_{2}}^{-1} .
\end{aligned}
$$

Then, according to (2.17) and (2.18),

$$
\frac{1}{\beta} \frac{\partial z_{R_{1} f_{1}}}{\partial æ_{R_{2} f_{2}}}=z_{f_{1} f_{2}}^{\prime}\left(R_{1} R_{2}\right)+\Delta_{f_{1} f_{2}}^{\prime}\left(R_{1} R_{2}\right) .
$$

Equation (3.2) can be written as

$$
\sum_{g \in R_{1}}\left[\delta_{f_{1} g}-P_{f_{1} g}\left(R_{1}\right) F_{f_{1} f_{1}}^{(1)}\left(R_{1}\right)\right] z_{g f_{2}}^{\prime}\left(R_{1} R_{2}\right)=-\Delta_{f_{1} f_{2}}^{\prime}\left(R_{1} R_{2}\right) .
$$

Relation analogous to (3.5) for the cluster $R_{1}^{\prime}$ which also contains the spin $f_{1}$ reads:

$$
\sum_{g \in R_{1}^{\prime}}\left[\delta_{f_{1} g}-P_{f_{1} g}\left(R_{1}^{\prime}\right) F_{f_{1} f_{1}}^{(1)}\left(R_{1}^{\prime}\right)\right] z_{g f_{2}}^{\prime}\left(R_{1}^{\prime} R_{2}\right)=-\Delta_{f_{1} f_{2}}^{\prime}\left(R_{1}^{\prime} R_{2}\right) .
$$

Let us now return to the uniform systems with (2.22) and (2.23); for the intracluster correlation functions $\eta_{f_{1} f_{2}}^{(2)}$ and $\eta_{R_{1} f_{1}}^{(1)}$ occurring in $P_{f_{1} f_{2}}\left(R_{1}\right)$ the relations $(2.25)$ and $(2.26)$ are obeyed, and the matrices $P_{f_{1} f_{2}}\left(R_{1}\right)$ and $P_{f_{1} f_{2}}\left(R_{1}^{\prime}\right)$ coincide. The $F_{f f^{\prime}}^{(4)}(R)$ matrix of DADP contains only even functions of $\eta_{q f}^{(1)}$ and $\eta_{q^{\prime} f}^{(1)}$; therefore, we can omit the factors $\mathrm{e}^{i \mathbf{k}_{Z} \mathbf{R}_{q}}$ and $\mathrm{e}^{i \mathbf{k}_{Z} \mathbf{R}_{q^{\prime}}}$. Adding equation (3.5) to (3.6), taking the factors $\mathrm{e}^{i \mathbf{k}_{Z} \mathbf{R}_{q}}$ and $\mathrm{e}^{i \mathbf{k}_{Z} \mathbf{R}_{q^{\prime}}}$. Adding equation (3.5) to (3.6), taking into account the relation

$$
b_{f_{1} f_{2}}\left(R_{1} R_{2}\right)=F_{f_{1} f_{1}}^{(1)}\left(R_{1}\right) z_{f_{1} f_{2}}^{\prime}\left(R_{1} R_{2}\right),
$$

which follows from (3.2), and the fact that

$$
-\left[\Delta_{f_{1} f_{2}}^{\prime}\left(R_{1} R_{2}\right)+\Delta_{f_{1} f_{1}}^{\prime}\left(R_{1}^{\prime} R_{2}\right)\right]=z_{f_{1} f_{2}}^{\prime}\left(R_{1} R_{2}\right)-\delta_{f_{1} f_{2}}\left(\delta_{R_{1} R_{2}}+\delta_{R_{1}^{\prime} R_{2}}\right),
$$

( $f_{1}$ is the common site of the clusters $R_{1}$ and $R_{1}^{\prime}$ ), we get

$$
\begin{aligned}
& -\delta_{f_{1} f_{2}}\left(\delta_{R_{1} R_{2}}+\delta_{R_{1}^{\prime} R_{2}}\right)= \\
& \quad=\sum_{R=R_{1}, R_{1}^{\prime}} \sum_{g \in R}^{\prime}\left\{\delta_{f_{1} g}\left[\frac{1}{F_{0}^{f, a}}-P_{f_{1} f_{1}}^{f, a}(R)\right]-P_{f_{1} g}^{f, a}(R)\right\} b_{g f_{2}}\left(R R_{2}\right),
\end{aligned}
$$


where in the sum $\sum_{R=R_{1}, R_{1}^{\prime}} \Sigma_{g \in R}^{\prime}$ the term with $g=f_{1}$ occurs only once. The matrix $\hat{P}^{f}\left(R_{1}\right)$ has the following elements

$$
\hat{P}^{f}\left(R_{1}\right)=\hat{P}^{f}\left(R_{1}^{\prime}\right)=\left(\begin{array}{cccc}
P_{0}^{f} & P_{1}^{f} & P_{2}^{f} & P_{1}^{f} \\
P_{1}^{f} & P_{0}^{f} & P_{1}^{f} & P_{2}^{f} \\
P_{2}^{f} & P_{1}^{f} & P_{0}^{f} & P_{1}^{f} \\
P_{1}^{f} & P_{2}^{f} & P_{1}^{f} & P_{0}^{f}
\end{array}\right),
$$

where

$$
\begin{aligned}
P_{0}^{f} & =\frac{F_{0}^{f}\left(F_{0}^{f}+F_{2}^{f}\right)-2\left(F_{1}^{f}\right)^{2}}{\left(F_{0}^{f}-F_{2}^{f}\right)\left(\left(F_{0}^{f}+F_{2}^{f}\right)^{2}-4\left(F_{1}^{f}\right)^{2}\right)}, \quad P_{1}^{f}=\frac{F_{1}^{f}}{\left(F_{0}^{f}+F_{2}^{f}\right)^{2}-4\left(F_{1}^{f}\right)^{2}}, \\
P_{2}^{f} & =\frac{2\left(F_{1}^{f}\right)^{2}-F_{2}^{f}\left(F_{0}^{f}+F_{2}^{f}\right)}{\left(F_{0}^{f}-F_{2}^{f}\right)\left(\left(F_{0}^{f}+F_{2}^{f}\right)^{2}-4\left(F_{1}^{f}\right)^{2}\right)},
\end{aligned}
$$

whereas

$$
\left[\hat{P}^{a}\left(R_{1}\right)\right]^{-1}=\left[\hat{P}^{a}\left(R_{1}^{\prime}\right)\right]^{-1}=\left(\begin{array}{cccc}
F_{0}^{a} & F_{1}^{a} & F_{2}^{a} & F_{3}^{a} \\
F_{1}^{a} & F_{0}^{a} & F_{3}^{a} & F_{2}^{a} \\
F_{2}^{a} & F_{3}^{a} & F_{0}^{a} & F_{1}^{a} \\
F_{3}^{a} & F_{2}^{a} & F_{1}^{a} & F_{0}^{a}
\end{array}\right) .
$$

It is convenient to Fourier transform equation (3.7) in positions vectors of cells $\mathbf{R}_{q}$ :

$$
\begin{aligned}
& P_{f g}(\mathbf{q}) \equiv \sum_{R} P_{f g}(R) \mathrm{e}^{-i \boldsymbol{q} \boldsymbol{R}_{q}} \mathrm{e}^{-i \boldsymbol{q}\left(\boldsymbol{r}_{f}-\boldsymbol{r}_{g}\right)} \\
& b_{f g}(\boldsymbol{q}) \equiv \sum_{R_{1}-R_{2}} b_{f g}\left(R_{1} R_{2}\right) \mathrm{e}^{-i \boldsymbol{q}\left(\boldsymbol{R}_{q_{1}}-\boldsymbol{R}_{q_{2}}\right)} \mathrm{e}^{-i \boldsymbol{q}\left(\boldsymbol{r}_{f}-\boldsymbol{r}_{g}\right)}
\end{aligned}
$$

Let us note that the sum in (3.10a) contains only two nonzero terms with $R=R_{1}$ and $R=R_{1}^{\prime}, f_{1}$ being the common site of the clusters $R_{1}$ and $R_{1}^{\prime}$. Then instead of (3.7), we get

$$
-\delta_{f_{1} f_{2}}=\sum_{g=1}^{4}\left\{\frac{\delta_{f_{1} g}}{F_{0}^{f, a}}-P_{f_{1} g}^{f, a}(\boldsymbol{q})\right\} b_{g f_{2}}(\boldsymbol{q})
$$

where

$$
\begin{aligned}
& P^{f, a}(\boldsymbol{q})=\left(\begin{array}{cccc}
2 P_{0}^{f, a} & P_{1}^{f, a}(\boldsymbol{q}) & P_{2}^{f, a}(\boldsymbol{q}) & P_{3}^{f, a}(\mathbf{q}) \\
P_{1}^{f, a}(\boldsymbol{q}) & 2 P_{0}^{f, a} & P_{4}^{f, a}(\boldsymbol{q}) & P_{5}^{f, a}(\boldsymbol{q}) \\
P_{2}^{f, a}(\boldsymbol{q}) & P_{4}^{f, a}(\boldsymbol{q}) & 2 P_{0}^{f, a} & P_{6}^{f, a}(\boldsymbol{q}) \\
P_{3}^{f, a}(\boldsymbol{q}) & P_{5}^{f, a}(\boldsymbol{q}) & P_{6}^{f, a}(\boldsymbol{q}) & 2 P_{0}^{f, a}
\end{array}\right) \\
& P_{0}^{f, a}=\frac{1}{1-\left(\eta^{(1)}\right)^{2}}, \\
& P_{1}^{f, a}(\boldsymbol{q})=2 P_{1}^{f, a} \cos \frac{q_{x}+q_{y}+q_{z}}{4}, \quad P_{2}^{f, a}(\boldsymbol{q})=2 P_{2}^{f, a} \cos \frac{q_{x}}{2}, \\
& P_{3}^{f}(\boldsymbol{q})=2 P_{1}^{f} \cos \frac{q_{x}-q_{y} \stackrel{4}{+} q_{z}}{4}, \quad P_{3}^{a}(\boldsymbol{q})=2 P_{3}^{a} \cos \frac{q_{x} \stackrel{2}{2} q_{y}+q_{z}}{4}, \\
& P_{4}^{f}(\boldsymbol{q})=2 P_{1}^{f} \cos \frac{q_{x}-\stackrel{4}{q}_{y}-q_{z}}{4}, \quad P_{4}^{a}(\boldsymbol{q})=2 P_{3}^{a} \cos \frac{q_{x}-{\stackrel{4}{q_{y}}-q_{z}}_{4}}{4}, \\
& P_{5}^{f, a}(\boldsymbol{q})=2 P_{2}^{f, a} \cos \frac{q_{y}}{2}, \\
& P_{6}^{f, a}(\boldsymbol{q})=2 P_{1}^{f, a} \cos \frac{q_{x}+q_{y}-q_{z}}{4},
\end{aligned}
$$


$\left(q_{x}, q_{y}, q_{z}\right.$ are projections of the dimensionless wavevector on the basic vectors of the tetragonal unit cell; $q_{i} \in[-\pi, \pi]$ ). Relations (3.11) comprise a close set of equations for the $\boldsymbol{q}$-dependent correlation functions of the reference systems corresponding to strained DKDP and DADP crystals.

\section{Dielectric permittivity}

Obtained in the previous section pair correlation function $b_{f_{1} f_{2}}(\boldsymbol{q})$ of the reference system enables one to calculate the correlation function of the general system $b_{f_{1} f_{2}}^{H}(\boldsymbol{q})$ related to the former by $(2.12)$, and hence the components of $\mathbf{q}$-dependent static dielectric susceptibilities of a clamped $\left(\varepsilon_{i}=\right.$ const) crystal. We need to calculate:

$$
\chi_{i j}(\boldsymbol{q}, T, p)=\frac{\beta}{4 v} \sum_{f f^{\prime}} \mu_{f}^{i} \mu_{f^{\prime}}^{j} b_{f f^{\prime}}^{H}(\boldsymbol{q}),
$$

where

$$
\begin{aligned}
& \mu_{1}=\mu_{3}^{1}=-\mu_{1}^{1}, \quad \mu_{2}^{1}=\mu_{4}^{1}=0, \\
& \mu_{2}=\mu_{2}^{2}=-\mu_{4}^{2}, \quad \mu_{1}^{2}=\mu_{3}^{2}=0, \\
& \mu_{3}=\mu_{1}^{3}=\mu_{2}^{3}=\mu_{3}^{3}=\mu_{4}^{3},
\end{aligned}
$$

are effective dipole moment of unit cells, assumed to be linear functions of pressure, and

$$
\hat{b}^{H}(\boldsymbol{q})=\left\{\hat{b}^{-1}(\boldsymbol{q})-\frac{\beta}{4} \hat{J}(\boldsymbol{q})\right\}^{-1}
$$

in the mean-field approximation used. With the help of the eigenfunctions $W_{f \mu}(\boldsymbol{q})$ and eigenvalues $b_{\mu}(\boldsymbol{q})$ of the $\hat{b}^{H}(\boldsymbol{q})$ matrix, we can get the following expressions for the transverse and longitudinal diagonal elements of the dielectric susceptibility tensor:

$$
\begin{aligned}
& \chi_{1}(\boldsymbol{q}, T, p)=\frac{\beta \mu_{1}^{2}}{v} \sum_{\mu} b_{\mu}(\boldsymbol{q})\left[W_{1 \mu}(\boldsymbol{q})-W_{3 \mu}(\boldsymbol{q})\right]^{2} ; \\
& \chi_{2}(\boldsymbol{q}, T, p)=\frac{\beta \mu_{2}^{2}}{v} \sum_{\mu} b_{\mu}(\boldsymbol{q})\left[W_{2 \mu}(\boldsymbol{q})-W_{4 \mu}(\boldsymbol{q})\right]^{2} ; \\
& \chi_{3}(\boldsymbol{q}, T, p)=\frac{\beta \mu_{3}^{2}}{v} \sum_{\mu} b_{\mu}(\boldsymbol{q})\left[\sum_{f} W_{f \mu}(\boldsymbol{q})\right]^{2} .
\end{aligned}
$$

Essential simplification occurs when the matrices $\hat{P}(\boldsymbol{q})$ (and thereby $\hat{b}^{-1}(\boldsymbol{q})$ ) and $\hat{J}(\boldsymbol{q})$ have the same symmetry. Then

$$
b_{\mu}(\boldsymbol{q})=\frac{1}{\tilde{P}_{\mu}(\boldsymbol{q})-\left[1-\left(\eta^{(1)}\right)^{2}\right]^{-1}-\beta \nu_{\mu}(\boldsymbol{q}) / 4},
$$

$\tilde{P}_{\mu}(\boldsymbol{q}), \nu_{\mu}(\boldsymbol{q})$ being the eigenvalues of $\hat{P}(\boldsymbol{q})$ and $\hat{J}(\boldsymbol{q})$ matrices.

In what follows we consider only the case of $\boldsymbol{q}=0$, looking for the expressions for the static susceptibilities of the crystals. It should be mentioned 
that due to the non-analytical properties of the $J(\boldsymbol{q})$ matrix at $q \rightarrow 0$, the values of susceptibilities in the center of Brillouin zone essentially depend on the direction along which the transition $q \rightarrow 0$ takes place, for instance, $\boldsymbol{q}=\left(0,0, q_{z} \rightarrow 0\right)$ and $\boldsymbol{q}=\left(q_{x} \rightarrow 0, q_{y} \rightarrow 0,0\right)$.

If we neglect the non-analytical properties of the $J(\boldsymbol{q})$ matrix, then

$$
W(0)=\frac{1}{2}\left(\begin{array}{cccc}
1 & 1 & 1 & 1 \\
1 & 1 & -1 & -1 \\
1 & -1 & 1 & -1 \\
1 & -1 & -1 & 1
\end{array}\right)
$$

is the common system of eigenvectors of $\hat{J}(\boldsymbol{q})$ and $\hat{P}(\boldsymbol{q})$ matrices, and

$$
\begin{aligned}
& \nu_{1,3}(0)=J_{11}(0)+J_{13}(0) \pm 2 J_{12}(0) ; \quad \frac{\nu_{1}(0)}{4}=\nu_{c}(0) \\
& \nu_{2,4}(0)=J_{11}(0)-J_{13}(0) ; \quad \frac{\nu_{2}(0)}{4}=\nu_{a}(0) ; \\
& \tilde{P}_{1,3}^{f}(0)=2\left(P_{0}^{f} \pm 2 P_{1}^{f}+P_{2}^{f}\right), \quad \tilde{P}_{1,3}^{a}(0)=2\left(P_{0}^{a}+P_{2}^{a} \pm\left(P_{1}^{a}+P_{3}^{a}\right)\right) \\
& \tilde{P}_{2,4}^{f}(0)=2\left(P_{0}^{f}-P_{2}^{f}\right), \quad \tilde{P}_{2,4}^{a}(0)=2\left(P_{0}^{a}-P_{2}^{a} \pm\left(P_{1}^{a}-P_{3}^{a}\right)\right)
\end{aligned}
$$

are their eigenvalues. Hence, we get the following expressions for the static susceptibilities of the crystals:

\section{DKDP}

$$
\begin{aligned}
& \chi_{1,2}^{\varepsilon}(0, T, p)=\frac{\beta \mu_{1,2}^{2}}{v} \frac{2 æ_{3}}{D^{f}-2 æ_{3} \varphi_{1}^{\eta}}, \\
& \chi_{3}^{\varepsilon}(0, T, p)=\frac{\beta \mu_{3}^{2}}{v} \frac{2 æ_{2}}{D^{f}-2 \varphi_{3}^{\eta} æ_{2}} ;
\end{aligned}
$$

$\underline{\text { DADP }}$

$$
\begin{aligned}
& \chi_{1,2}^{\varepsilon}(0, T, p)=\frac{\beta \mu_{1,2}^{2}}{2 v}\left[\frac{2 æ_{1}}{D^{a}-2 æ_{1} \varphi_{1}^{\eta}}+\frac{2 æ_{2}}{D^{a}-2 æ_{2} \varphi_{1}^{\eta}}\right], \\
& \chi_{3}^{\varepsilon}(0, T, p)=\frac{\beta \mu_{3}^{2}}{v} \frac{2 æ_{3}}{D^{a}-2 æ_{3} \varphi_{3}^{\eta}} ;
\end{aligned}
$$

Here we introduce the notations:

$$
æ_{1}=1+b \cosh z, æ_{2}=\cosh 2 z+b \cosh z-\left[\eta^{(1)}\right]^{2} D, æ_{3}=a+b \cosh z,
$$

and

$$
\varphi_{1}^{\eta}=\frac{1}{1-\left[\eta^{(1)}\right]^{2}}+\beta \nu_{a}(0), \quad \varphi_{3}^{\eta}=\frac{1}{1-\left[\eta^{(1)}\right]^{2}}+\beta \nu_{c}(0) .
$$

Let us note that the expressions for the dielectric susceptibilities coincide with those obtained in [1-5] by means of a different method.

Dielectric permittivity is related to susceptibility as

$$
\varepsilon_{i}^{\varepsilon}(0, T, p)=\varepsilon_{i \infty}+4 \pi \chi_{i}^{\varepsilon}(0, T, p) .
$$

Since the values of the piezoelectric constants $d_{i j}$ of a DADP crystal are fairly large, to compare our results with the experimental data, we need 
to calculate the dielectric permittivities of a free crystal $\varepsilon_{i}^{F}(0, T, p)$, which are usually measured. The "free" permittivities of DADP are related to "clamped" ones by the formulas

$$
\begin{aligned}
& \varepsilon_{1}^{F}(0, T, p)=\varepsilon_{1}^{\varepsilon}(0, T, p)+4 \pi \frac{d_{14}^{2}}{s_{44}^{E}}, \\
& \varepsilon_{2}^{F}(0, T, p)=\varepsilon_{2}^{\varepsilon}(0, T, p)+4 \pi \frac{d_{25}^{2}}{s_{55}^{E}}, \\
& \varepsilon_{3}^{F}(0, T, p)=\varepsilon_{3}^{\varepsilon}(0, T, p)+4 \pi \frac{d_{36}^{2}}{s_{66}^{E}},
\end{aligned}
$$

where $d_{i j}$ are the piezoelectric constants, and $s^{E}$ are the elastic shear compliances of a short circuited crystal [20]. In case of DKDP the difference between permittivities of clamped and free crystal is negligibly small.

\section{Numerical analysis}

The formulas for the $\boldsymbol{q}$-dependent correlation functions and dielectric permittivities as well as the systems of equations for the order parameters and strains (2.30) and (2.31) contain the following fitting parameters

- $\varepsilon^{0}, w^{0}, w_{1}^{0} \rightarrow \infty, \nu_{c}^{0}(0), f_{1}^{0)}=\left(\mu_{1}^{(0)}\right)^{2} / v, f_{3}^{0)}=\left(\mu_{3}^{(0)}\right)^{2} / v$ for both DKDP and DADP, $\nu_{a}^{0}\left(\mathbf{k}_{Z}\right), \nu_{a}^{0}(0)$ for DADP - relevant for unstrained crystals;

- $\delta_{1 i}, \delta_{2 i}, \psi_{c i}(0), \delta_{1} / \delta_{0}, \partial \mu_{1} / \partial p, \partial \mu_{3} / \partial p$ for DKDP and DADP, $\psi_{a i}\left(\mathbf{k}_{Z}\right), \psi_{a i}(0)$ for DADP - the so-called deformation potentials, and

- $c_{i j}^{(0)}$ - the "seed" elastic constants.

Their values, chosen as described below are given in Tables 1, 2, 3 .

The values of $\varepsilon^{0}, w^{0}, \nu_{c}^{0}(0)$ for DKDP and $\nu_{a}\left(\mathbf{k}_{Z}\right)$ for DADP have been found in $[6,15,16]$. They provide a satisfactory description of the temperature dependences of static and dynamic dielectric permittivities, spontaneous polarization, specific heat at ambient pressure as well as values of transition temperatures of the crystals.

The values of the deformation potentials $\delta_{1 i}$ of DKDP and DADP, $\psi_{c i}(0)$ of DKDP and $\psi_{a i}\left(\mathbf{k}_{Z}\right)$ of DADP have been chosen in [1-5]. They yield a good fit to the available experimental data for variation of transition temperature, spontaneous polarization, longitudinal dielectric permittivity of DKDP and transition temperature of DADP with hydrostatic pressure. The values of $\delta_{2 i}$ parameters were determined from the criteria (2.34) and (2.35). The dependence of the D-site distance $\delta$ on hydrostatic pressure in DKDP has been reported in $[13,14]$. The value of $\delta_{1} / \delta_{0}$ in the case of DADP was found from the available data for the $T_{\mathrm{N}}(\delta)$ and $T_{\mathrm{N}}(p)$ dependences $[17,18]$. In the case of the uniaxial pressure $\sigma_{3}$, the $\delta_{1} / \delta_{0}$ parameter was chosen such that a good fit to the reported in [4] $T_{\mathrm{C}}\left(\sigma_{3}\right)$ dependence was obtained. Let us mention that the transition temperature $T_{\mathrm{C}}\left(T_{\mathrm{N}}\right)$ decreases with the uniaxial pressure $\sigma_{3}$ several times more rapidly that with hydrostatic $\sigma_{\mathrm{h}}$ : 


\section{$\underline{\mathrm{DKDP}}$}

$$
\begin{aligned}
& \delta_{1} / \delta_{0}\left(\sigma_{\mathrm{h}}\right)=-0.89 \cdot 10^{-5} \mathrm{bar}^{-1}, \quad \delta_{1} / \delta_{0}\left(\sigma_{3}\right)=-0.7 \cdot 10^{-4} \mathrm{bar}^{-1}, \\
& \frac{\partial T_{\mathrm{C}}}{\partial p}\left(\sigma_{\mathrm{h}}\right)=-2.67 \mathrm{~K} / \mathrm{kbar}, \quad \frac{\partial T_{\mathrm{C}}}{\partial p}\left(\sigma_{3}\right)=-13.9 \mathrm{~K} / \mathrm{kbar},
\end{aligned}
$$

$\underline{\mathrm{DADP}}$

$$
\begin{aligned}
& \delta_{1} / \delta_{0}\left(\sigma_{\mathrm{h}}\right)=-3.35 \cdot 10^{-6} \mathrm{bar}^{-1}, \quad \delta_{1} / \delta_{0}\left(\sigma_{3}\right)=-1.75 \cdot 10^{-5} \mathrm{bar}^{-1}, \\
& \frac{\partial T_{\mathrm{N}}}{\partial p}\left(\sigma_{\mathrm{h}}\right)=-1.4 \mathrm{~K} / \mathrm{kbar}, \quad \frac{\partial T_{\mathrm{N}}}{\partial p}\left(\sigma_{3}\right)=-9.0 \mathrm{~K} / \mathrm{kbar} .
\end{aligned}
$$

The values of $\psi_{a i}(0)$ and $\psi_{c i}(0)$ for DADP crystal hardly affect the dielectric responses of the crystals, so we put them equal to zero. The chosen value of derivative $\partial \mu_{3} / \partial p$ provides a good description of the dependences of spontaneous polarization and longitudinal static dielectric permittivity on hydrostatic pressure in the case of DKDP. As far as $\partial \mu_{1} / \partial p$ in DKDP and both $\partial \mu_{3} / \partial p$ and $\partial \mu_{1} / \partial p$ in DADP crystals are concerned, no appropriate experimental data are available to determine their values. The values of the elastic constants of paraelectric DKDP and DADP crystals were reported in $[19,20]$. Those values were taken as "seed" elastic constants $c_{i j}^{(0)}$.

Further experimental studies of hydrostatic and uniaxial pressure effects are required to ascertain the values of the theory parameters.

Table 1 . The theory parameters (in K) for unstrained crystals.

\begin{tabular}{|l|c|c|c|c|c|c|}
\hline & $T_{\mathrm{C}}$ & $\varepsilon^{0}$ & $w^{0}$ & $\nu_{a}^{0}\left(\mathbf{k}_{Z}\right)$ & $\nu_{a}^{0}(0)$ & $\nu_{c}^{0}(0)$ \\
\hline DKDP & 210 & 87.5 & 785 & & & 36.8 \\
DADP & 235 & 77 & 709 & 85.5 & -54 & -17 \\
\hline
\end{tabular}

Table 2. The deformation potentials (in K) for DKDP [1-4] and DADP $[5,6]$ crystals; $\mathbf{k}=0$ for DKDP and $\mathbf{k}=\mathbf{k}_{Z}$ for DADP.

\begin{tabular}{|l|ccccc|ccccc|}
\hline & $\delta_{11}^{-}$ & $\delta_{12}^{-}$ & $\delta_{13}^{-}$ & $\delta_{11}^{+}$ & $\delta_{13}^{+}$ & $\psi_{1}^{-}(\mathbf{k})$ & $\psi_{2}^{-}(\mathbf{k})$ & $\psi_{3}^{-}(\mathbf{k})$ & $\psi_{1}^{+}(\mathbf{k})$ & $\psi_{3}^{+}(\mathbf{k})$ \\
\hline DKDP & -45 & -55 & 885 & -50 & 800 & -85 & -105 & 250 & -95 & 390 \\
DADP & -38 & -46 & 741 & -42 & 665 & -180 & -252 & 112 & -239 & 109 \\
\hline
\end{tabular}

Table 3. The "seed" elastic constants (units of $10^{11} \mathrm{dyn} / \mathrm{cm}^{2}$ ) of a DKDP [19] and DADP [20] crystals.

\begin{tabular}{|l|cccccccccc|}
\hline & $c_{11}^{0+}$ & $c_{12}^{0+}$ & $c_{13}^{0+}$ & $c_{33}^{0+}$ & $c_{11}^{0-}$ & $c_{12}^{0-}$ & $c_{13}^{0-}$ & $c_{22}^{0-}$ & $c_{23}^{0-}$ & $c_{23}^{0-}$ \\
\hline DKDP & 6.93 & -0.78 & 1.22 & 5.45 & 6.93 & -1.78 & 1.22 & 6.0 & 1.1 & 5.0 \\
DADP & 6.59 & 0.53 & 1.92 & 3.28 & 6.24 & 0.4 & 1.66 & 6.4 & 1.5 & 3.48 \\
\hline
\end{tabular}

In figure 1 we depict the wavevector dependences of some pair correlation functions of a DKDP crystal at different values of hydrostatic pressure and temperature. 

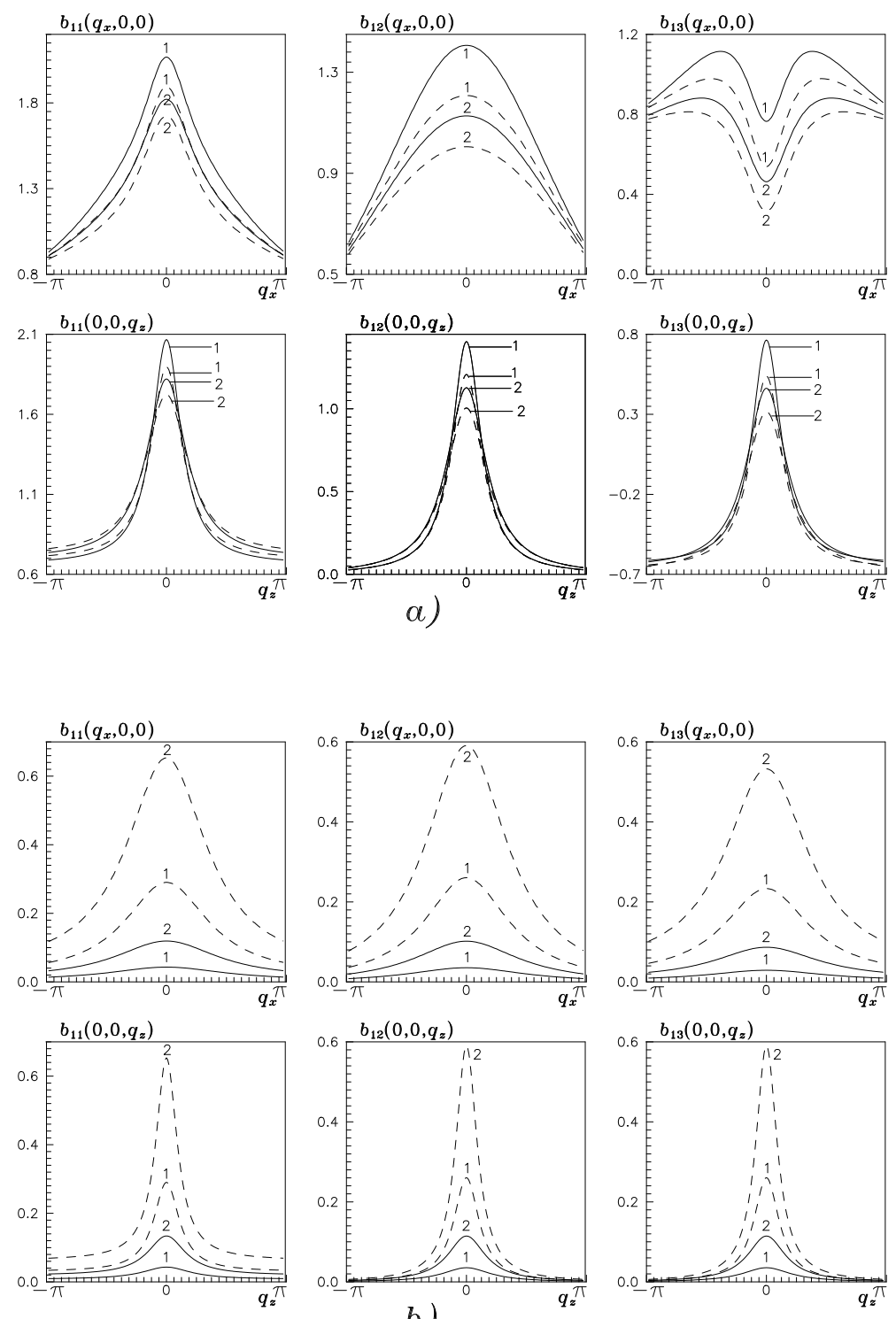

b)

Figure 1. The deuteron pair correlation functions of a DKDP crystal as functions of wavevector at different values of temperature $T(\mathrm{~K})$ : a) 1 $190,2-200$; b) $1-220 ; 2-250$ and hydrostatic pressure $p$ (kbar): solid lines -0.001 , dashed lines -10 . 
As one can see, $b_{11}(\boldsymbol{q})>b_{12}(\boldsymbol{q})>b_{13}(\boldsymbol{q})$. Overall, the correlation functions have maxima in the center of the Brillouin zone, except for the $b_{11}(q, 0,0)$ which has a minimum at $q=0$. The amplitude of spin correlations increases when temperature approaches $T_{\mathrm{C}}$. Therefore, an increase in temperature and increase in pressure in the paraelectric phase and decrease in temperature and decrease in pressure in the ferroelectric phase influence the correlations in a similar way, because all of them move the system from the transition point. A strong anisotropy of correlation functions is observed along the $(q, 0,0)$ and $(0,0, q)$ directions; that agrees with the result of [11].

In figure 2 we plot the wavevector dependences of the pair correlation functions of a DADP crystal at different values of hydrostatic pressure and temperature at $T>T_{\mathrm{N}}$. Here, also $b_{11}(\boldsymbol{q})>b_{12}(\boldsymbol{q})>b_{13}(\boldsymbol{q})$. However, the maxima of $b_{12}(q, 0,0)$ and $b_{13}(q, 0,0)$ shift from the center to the boundary of Brillouin zone. Furthermore, $b_{12}(\boldsymbol{q})$ and $b_{13}(\boldsymbol{q})$ increase with pressure and temperature.
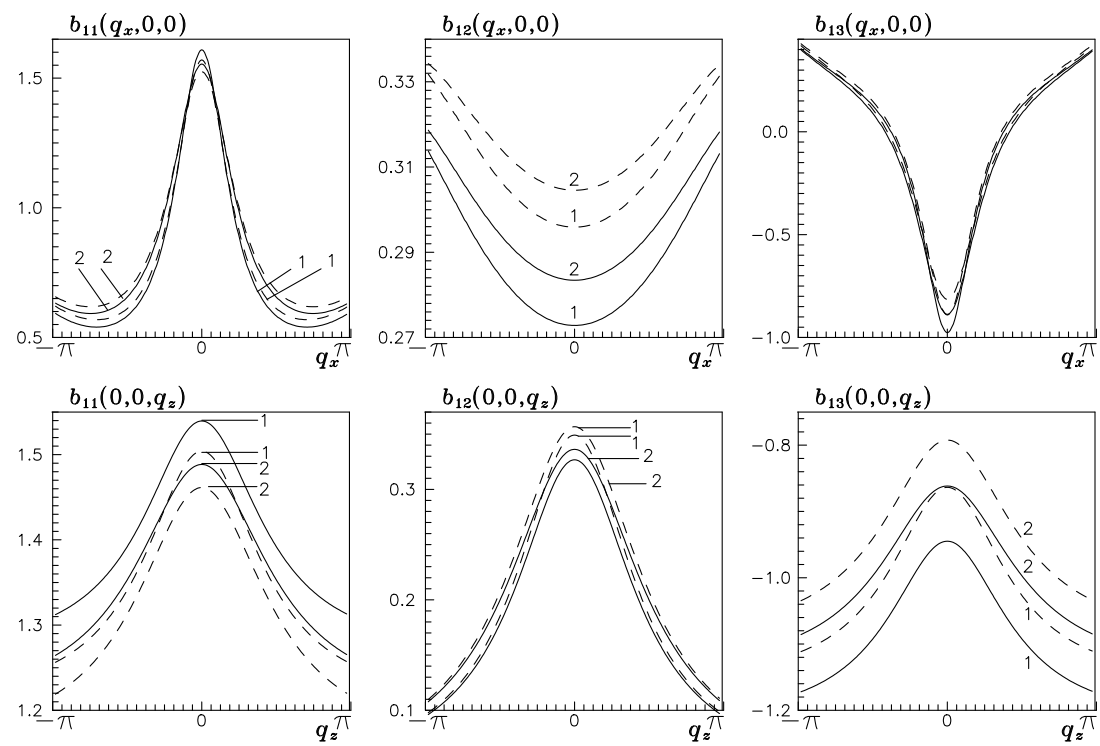

Figure 2. The deuteron pair correlation functions of a DADP crystal as functions of wavevector at different values of temperature $T(\mathrm{~K}): 1-240$, $2-270$ and hydrostatic pressure $p(\mathrm{kbar})$ : solid lines -0.001 , dashed lines -10 .

The temperature dependence of the inverse longitudinal dielectric permittivity $\varepsilon_{3}^{-1}(0, T, p)$ of a DKDP crystal at different hydrostatic and uniaxial $-p=\sigma_{3}$ pressures is plotted in figures 3 and 4 , respectively. In figure 4 we also depicted the $\varepsilon_{3}^{-1}(0, T, p)$ curve at hydrostatic pressure of $1 \mathrm{kbar}$.

As can be seen, in the paraelectric phase the Curie-Weiss law is obeyed in a wide temperature range at different pressures, the Curie constant decreasing with hydrostatic pressure and being independent of uniaxial pressure. The main effect of the uniaxial pressure is the shift of the transition point and, thereby, of the whole $\varepsilon_{3}(T)$ curve to lower temperatures. Therefore, at constant $\Delta T$, the $\varepsilon_{3}(0, T, p)$ almost does not depend on pressure in the paraelectric phase and only slightly varies in the ferroelectric phase. 


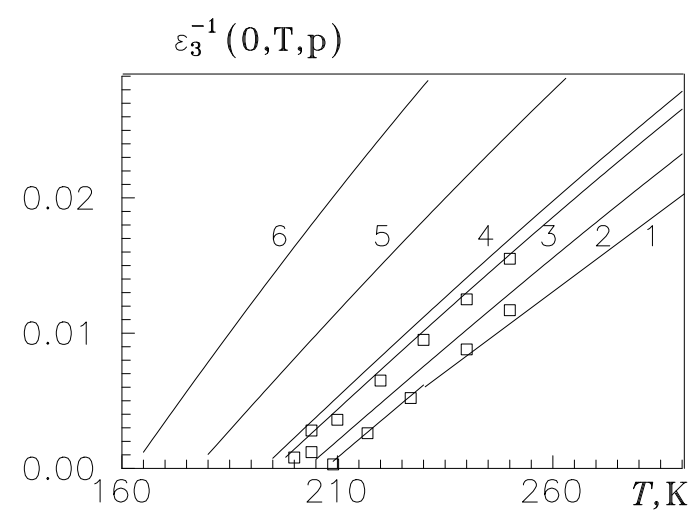

Figure 3. The inverse longitudinal dielectric permittivity of a $\mathrm{KD}_{2} \mathrm{PO}_{4}$ crystal as a function of temperature at values of hydrostatic pressure $p(\mathrm{kbar})$ : $1-0.001 ; 2-1.8 ; 3-4.14 ; 4-5.0 ; 5-10.0 ; 6-15.0$. The experimental points are taken from [21].

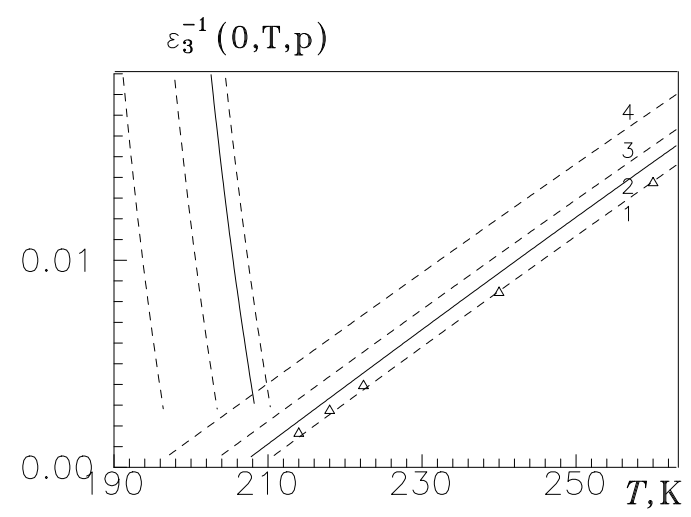

Figure 4 . The temperature dependence of the inverse longitudinal static dielectric permittivity $\varepsilon_{3}^{-1}(0, T, p)$ of a DKDP crystal at different values of uniaxial $\sigma_{3}$ (dashed lines) pressure $p$ (kbar): $1, \triangle[22],-0.001 ; 2,4$ $-1 ; 3-0.5$. The solid line corresponds to hydrostatic pressure of 1 kbar.

In figures 5 and 6 we plot the calculated temperature dependences of the static transverse $\varepsilon_{1}(0, T, p)$ and longitudinal $\varepsilon_{3}(0, T, p)$ permittivities of a DADP crystal at different pressures along with the experimental points for the ambient pressure. We are not aware of any experimental measurement of pressure effects on dielectric characteristics of deuterated DADP.

Theoretical values of "free" permittivities are calculated using relations (4.16) and experimental data for appropriate piezoelectric constants and elastic shear compliances [20]. Since in DADP crystal $d_{36} \gg d_{14}$, the difference between clamped and free values of the longitudinal permittivity is several times larger than that of transverse permittivity. A good description of experimental data is obtained for $\varepsilon_{1}(0, T, p)$, while for $\varepsilon_{3}(0, T, p)$ a small discrepancy between theory and experiment is observed. Probably, 
we could remove the discrepancy by explicit taking into account of shear strain $\varepsilon_{6}$.

The main pressure effect is in the shift of the $\varepsilon_{i}(0, T, p)$ curves to lower temperatures. Besides, $\varepsilon_{1}(0, T, p)$ and $\varepsilon_{3}(0, T, p)$ of DADP decrease with pressure in the paraelectric phase and increase in the antiferroelectric phase, with the pressure effect being much stronger at $T>T_{\mathrm{N}}$.
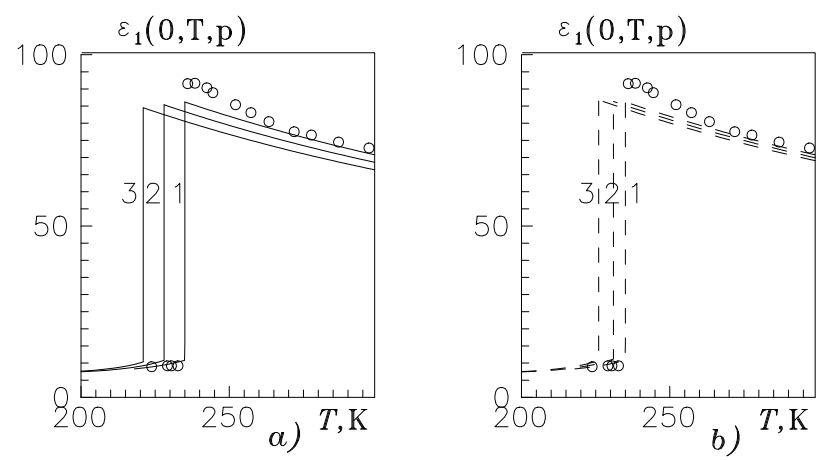

Figure 5. The temperature dependence of the transverse static dielectric permittivity of an $\mathrm{ND}_{4} \mathrm{D}_{2} \mathrm{PO}_{4}$ crystal at different pressures $p(\mathrm{kbar})$ : a) (hydrostatic) $1, \circ[20]-0.001 ; 2-5 ; 3-10 ;$ b) (uniaxial $\left.\sigma_{3}\right) 1$, ० [20] $-0.001 ; 2-0.5 ; 3-1$.
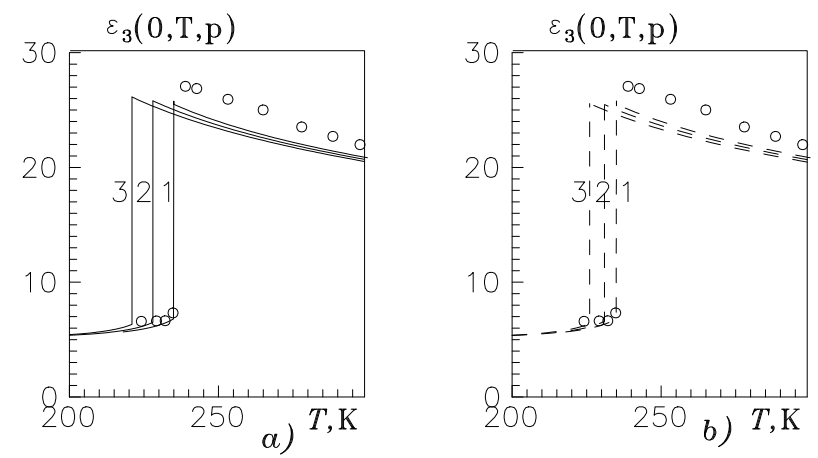

Figure 6. The temperature dependence of the longitudinal static dielectric permittivity of an $\mathrm{ND}_{4} \mathrm{D}_{2} \mathrm{PO}_{4}$ crystal at different pressures $p$ (kbar): a) (hydrostatic) $1, \circ[20]-0.001 ; 2-5 ; 3-10$; b) (uniaxial $\left.\sigma_{3}\right) 1$, ० $[20]-0.001 ; 2-0.5 ; 3-1$.

\section{Conclusions}

In this paper, on the basis of previously proposed model [1-6], we study the influence of external hydrostatic and uniaxial $\sigma_{3}$ pressure on the $\boldsymbol{q}$ dependent pair correlation functions and dielectric properties of DKDP and DADP crystals. In the four-particle cluster approximation, we derive an 
equation for these functions and find expressions for static dielectric permittivities of these crystals as functions of pressure and temperature. Theoretical results are compared with available experimental data. It is shown that under the proper choice of fitting parameters, the theory provides a satisfactory description of variation of studied characteristics with hydrostatic pressure.

We also state the possible changes in the dielectric properties of DKDP and DADP crystals with the uniaxial pressure, assuming some plausible changes in the crystal structure with pressure. The main feature of the predicted effects is that even low uniaxial stress can induce a significant shift of the transition temperature, whereas the intrinsic changes in the responses of DKDP and DADP crystals (i.e. beyond the shift) are rather small.

It is necessary to carry out comprehensive experimental studies of the uniaxial pressure effects on these crystals, especially on their structure. We hope that these measurements will allow us to define the theory parameters more precisely and verify our predictions.

\section{References}

[1] Stasyuk I.V., Levitskii R.R., Zachek I.R., Moina A.P., Hydrostatic and uniaxial pressure effects on phase transition and physical properties of $\mathrm{KD}_{2} \mathrm{PO}_{4}{ }^{-}$ type ferroelectrics. Four-particle cluster approximation. // Submitted to Mol. Phys. Reports.

[2] Stasyuk I.V., Levitskii R.R., Zachek I.R., Moina A.P., Duda A.S. Hydrostatic pressure influence on phase transition and physical properties of $\mathrm{KD}_{2} \mathrm{PO}_{4}-$ type ferroelectrics. // Cond. Matt. Phys., 1996, No 8, p. 129-156.

[3] Stasyuk I.V., Levitskii R.R., Zachek I.R , Moina A.P., Duda A.S. Hydrostatic pressure influence on phase transition and physical properties of $\mathrm{KD}_{2} \mathrm{PO}_{4}{ }^{-}$ type ferroelectrics. / Preprint ICMP-96-12E, Lviv, 1996, 42 p.

[4] Stasyuk I.V., Levitskii R.R., Zachek I.R., Duda A.S., Moina A.P., Romanyuk N.O., Stadnyk V.J., Chervony R.G., Shcherbina Ye.V. Uniaxial pressure influence on phase transition and physical properties of highly deuterated $\mathrm{K}\left(\mathrm{H}_{x} \mathrm{D}_{1-x}\right)_{2} \mathrm{PO}_{4}$-type ferroelectrics./ Preprint, ICMP-96-18E, Lviv, 1996, 36 $\mathrm{p}$.

[5] Levitskii R.R., Moina A.P., Zachek I.R. External pressure influence on phase transition and physical properties of $\mathrm{ND}_{4} \mathrm{D}_{2} \mathrm{PO}_{4}$-type antiferroelectrics. / Preprint, ICMP-97-3E, Lviv, 1997, 36 p.

[6] Levitskii R.R., Zachek I.R., Moina A.P., Mits Ye.V., External pressure influence on phase transition and physical properties of $\mathrm{ND}_{4} \mathrm{D}_{2} \mathrm{PO}_{4}$-type antiferroelectrics. To be published.

[7] Stasyuk I.V., Biletskii I.N. Influence of omnidirectional and uniaxial stress on the ferroelectric phase transition in crystals of $\mathrm{KH}_{2} \mathrm{PO}_{4}$ type. // Bull. Ac.Sci.USSR. Phys.Ser., 1983, vol. 4, No 4, p. 79-82.

[8] Stasyuk I.V., Biletskii I.N., Styahar O.N. Pressure induced pressure phase transition in $\mathrm{KD}_{2} \mathrm{PO}_{4}$ crystals. // Ukr. Fiz. Zh., 1986, vol. 31, No 4, p. 567571 (in Russian).

[9] Lovesey S.W., Marshall W. Theory of thermal neutron scattering. Oxford, Clarendon Press, 1971.

[10] Levitskii R.R., Korinevskii N.A., Stasyuk I.V. Distribution functions and thermodynamical properties of $\mathrm{KD}_{2} \mathrm{PO}_{4}$ and $\mathrm{ND}_{4} \mathrm{D}_{2} \mathrm{PO}_{4}$ type crystals. // Phys. Stat. Solidi (b), 1978, vol. 88, No 1, p. 51-63.

[11] Havlin S. Sompolinsky H. Cluster approximation for $q$-dependent correlations on magnetic and ferroelectric systems. // Phys. Rev. B, vol. 25, No 9, p. $5828-5835$.

[12] Levitskii R.R., Sorokov S.I. Investigations of the Ising-type models within cluster approach. // Cond. Matt. Phys., vol. 3, p. 79-115. 
[13] Nelmes R.J. Structural studies of KDP and the KDP-type transition by neutron and X-ray diffraction: 1970-1985. // Ferroelectrics, 1987, vol. 71, p. $87-123$.

[14] Tibbals J.E., Nelmes R.J., McIntyre G.J. The crystal structure of tetragonal $\mathrm{KH}_{2} \mathrm{PO}_{4}$ and $\mathrm{KD}_{2} \mathrm{PO}_{4}$ as a function of temperature and pressure. // J.Phys.C: Solid State. Phys., 1982, vol. 15, p. 37-58.

[15] Levitskii R.R., Zachek I.R., Mits Ye.V. Thermodynamics and longitudinal relaxation of ferroelectrics $\mathrm{K}\left(\mathrm{H}_{x} \mathrm{D}_{1-x}\right)_{2} \mathrm{PO}_{4} /$ Preprint, ITP-87-114R, Kiev, 1987, 48 p (in Russian).

[16] Levitskii R.R., Zachek I.R., Mits Ye.V. Transverse relaxation in $\mathrm{K}\left(\mathrm{H}_{x} \mathrm{D}_{1-x}\right)_{2} \mathrm{PO}_{4}$ type ferroelectrics. / Preprint, ITP-87-115R, Kiev, 1987, 48 $\mathrm{p}$ (in Russian).

[17] Skalyo J., Jr., Frazer B.C., Shirane G., Daniels W.B. The pressure dependence of the transition temperature in KDP and ADP. // J. Phys. Chem. Solids, 1969, vol. 30, p. 2045-2051.

[18] McMahon M.I., Piltz R.O., Nelmes R.J. Neutron-diffraction studies of the relationship between $T_{\mathrm{C}}$ and $\mathrm{H}$-bond dimensions in $\mathrm{H}$-ordering transitions. // Ferroelectrics, 1990, vol. 108, p. 277-282.

[19] Shuvalov L.A., Mnatsakanyan A.V. The elastic properties of $\mathrm{KD}_{2} \mathrm{PO}_{4}$ crystals over a wide temperature range. // Sov. Phys. Crystall., 1966, vol. 11, No 2, p. $210-212$.

[20] Mason W.P., Matthias B.T. The piezoelectric, dielectric and elastic properties of $\mathrm{ND}_{4} \mathrm{D}_{2} \mathrm{PO}_{4}$ (deuterated ADP). // Phys. Rev., 1952, vol. 88, No 3, p. 477479.

[21] Samara G.A. Pressure dependence of the static dielectric properties of $\mathrm{KD}_{2} \mathrm{PO}_{4}$ and $\mathrm{RbH}_{2} \mathrm{PO}_{4}$. // Ferroelectrics, 1979, vol. 22, p. 925-936.

[22] Hill R.M., Ichiki S.K. Paraelectric response of $\mathrm{KD}_{2} \mathrm{PO}_{4}$. // Phys. Rev., 1963, vol. 130, No 1, p. 150-151.

\title{
q-ЗАЛЕЖНІ КОРЕЛЯЩІЙНІ ФУНКЦІї І ДІЕЛЕКТРИЧНІ ПРОНИКЛИВОСТІ КРИСТАЛІВ ТИПУ DKDP I DADP. ВПЛИВ ЗОВНІШНЬОГО ТИСКУ
}

\begin{abstract}
А.П.Моїна
В наближенні чотиричастинкового кластера отримано рівняння для $\boldsymbol{q}$-залежних парних кореляційних функцій дейтронів кристалів DKDP i DADP, до яких пркладено зовнішній гідростатичний чи одновісний $\sigma_{3}$ тиск. Досліджуються іх залежності від хвильового вектора, температури та тиску. Знайдено вирази для статичних діелектричних проникливостей деформованих кристалів, що розглядаються. Показано, що при належному виборі параметрів теорії, отримані результати задовільно узгоджуються з наявними експериментальними даними.
\end{abstract}

